\title{
Multi-slice passband bSSFP for human and rodent fMRI at ultra-high field
}

\author{
Olivier Reynaud, Analina R. da Silva, Rolf Gruetter, Ileana O. Jelescu* \\ Centre d'Imagerie Biomédicale, Ecole Polytechnique Fédérale de Lausanne, Lausanne, Switzerland
}

\section{A R T I C L E I N F O}

\section{Article history:}

Received 7 January 2019

Revised 7 May 2019

Accepted 28 May 2019

Available online 30 May 2019

\section{Keywords:}

Balanced SSFP

BOLD

fMRI

Ultra-high field

Multi-slice

\begin{abstract}
A B S T R A C T
Balanced steady-state free precession (bSSFP) can be used as an alternative to gradient-echo (GE) EPI for BOLD functional MRI when image distortions and signal drop-outs are severe such as at ultra-high field. However, 3D-bSSFP acquisitions have distinct drawbacks on either human or animal MR systems. On clinical scanners, 3D imaging is suboptimal for localized fMRI applications. It can also display distortions when acceleration methods such as spiral read-outs are used, and, compared to multi-slice acquisitions, suffers from increased sensitivity to motion or physiological noise which further results in blurring. On pre-clinical systems, 3D acquisitions have low temporal resolution due to limited acceleration options, while single slice often results in insufficient coverage. The aim of the present study was to implement a multi-slice bSSFP acquisition with Cartesian read-out to obtain non-distorted BOLD fMRI activation maps in the human and rat brain at ultra-high field. We show that, when using a new pseudo-steadystate, the bSSFP signal characteristics are preserved. In the human brain at $7 \mathrm{~T}$, we demonstrate that both task- and resting-state fMRI can be performed with multi-slice bSSFP, with a temporal SNR that matches that of 3D-bSSFP, resulting in - at least - equal performance. In the rat brain at $14 \mathrm{~T}$, we show that the multi-slice bSSFP protocol has similar sensitivity to gradient-echo EPI for task fMRI, while benefitting from much reduced distortions and drop-outs. The advantages of passband bSSFP at $14 \mathrm{~T}$ in comparison with GE-EPI are expected to be even more marked for mouse brain.
\end{abstract}

(c) 2019 Elsevier Inc. All rights reserved.

\section{Introduction}

The most widespread imaging sequence used in functional MRI (fMRI) is gradient-echo echo planar imaging (GE-EPI). This sequence offers good sensitivity to the blood oxygenation level dependent (BOLD) signal via its $T_{2}^{*}$ contrast, while allowing a high temporal resolution. However, at ultra-high field, GE-EPI suffers from two significant drawbacks related to $B_{0}$ inhomogeneity: spatial distortions making registration to an anatomical reference difficult, and, more importantly, significant signal loss in areas of interest due to susceptibility mismatches close to air-tissue interfaces.

On the other hand, it has been reported that balanced steadystate free precession (bSSFP) can measure a significant BOLD effect

\footnotetext{
Abbreviations: BOLD, blood oxygenation level dependent; bSSFP, balanced steady-state free precession; EPI, echo planar imaging; fMRI, functional magnetic resonance imaging; GE, gradient echo; SNR, signal-to-noise ratio.

* Corresponding author at: Centre d'Imagerie Biomédicale, EPFL ENT-R CIBM-AIT, CH F1 626 (Bâtiment CH), Station 6, CH-1015 Lausanne, Switzerland.

E-mail address: ileana.jelescu@epfl.ch (I.O. Jelescu).
}

[1] while offering two key advantages at ultra-high field: it has the highest signal-to-noise ratio (SNR) efficiency of all known pulse sequences, and exhibits minimal susceptibility-related distortions and signal loss [2]. Initially, bSSFP fMRI experiments were performed in the transition-band regime, where indeed dramatic signal magnitude and phase changes were observed with activation $[3,4]$. However, this method is demanding in terms of shimming and offers limited brain coverage for a given phase cycling angle. Passband bSSFP does not suffer from these drawbacks and has also been shown to display excellent BOLD sensitivity, comparable to that of GE-EPI [1] and superior to TR-matched Cartesian GE [5,6].

The sources of BOLD contrast in passband bSSFP are multiple and their relative weight depends on sequence parameters and field strength. At short TE/TR, the BOLD contrast is largely due to $T_{2}$ changes (i.e. non-refocusable dephasing due to diffusion in an inhomogeneous field), while increasing the TE/TR will introduce more $T_{2}^{*}$ weighting, similar to GE [5,7-9]. While most spins will remain within the broad passband of the bSSFP profile during rest and activation, some contribution to signal change from frequency variations (similar to those that dominate in transition band bSSFP) 
can also contribute $[6,10]$, especially at ultra-high field where intra-voxel frequency distributions are broader.

These sources of contrast also impact the spatial specificity of the bSSFP BOLD signal: a short TR increases the relative contribution of extravascular signal around capillaries rather than larger veins and improves specificity. However, a short TR implies that intravascular spins - whether in capillaries or larger veins - will also contribute to the BOLD signal. Previous simulation work, validated by experiments at $3 \mathrm{~T}$, calculated that the intravascular contribution represented indeed a large fraction of the bSSFP fMRI contrast, but high field and short TR have the potential to provide a localization of the activation closer to the capillaries $[8,11]$. Another study at $9.4 \mathrm{~T}$ in rats [6] reported a spatial shift in the activation foci with different phase cycling angles in the bSSFP acquisitions, which implied that spins contributing to the BOLD contrast may stem from different pools depending on the spatial location and on the magnetic field inhomogeneities.

The requirement to maintain a steady-state implies the use of bSSFP as a 3D technique. However, the need for high temporal resolution in fMRI combines poorly with Cartesian 3D acquisitions, particularly in rodent imaging where acceleration options are very limited. Aside from a recent implementation of compressed sensing for bSSFP fMRI in the rat at 9.4 T [12], rodent studies so far have been limited to single slice acquisitions [6,13-15]. The latter preclude sufficient coverage to effectively describe entire functional regions or large-scale resting-state networks. One study used multi-slice bSSFP to examine the mouse auditory pathway but did not report how the departure from steady-state was handled and whether the frequency offset profile was centered on a flat passband [16].

On clinical MR systems, 3D bSSFP is typically accelerated using radial [17], spiral [18], or multi-line read-outs [19], as well as parallel imaging [20]. However, 3D acquisitions can be sub-optimal for specific applications such as brainstem or retina fMRI, due to increased sensitivity to motion and physiological noise [5] and blurring along the second phase-encode direction.

Therefore, the aim of the present study was to implement a multi-slice passband bSSFP in the transient state for BOLD fMRI at ultra-high field ( $7 \mathrm{~T}$ for human experiments and $14 \mathrm{~T}$ for rat experiments). The use of bSSFP in the transient state has been proposed previously for X-nuclei applications [21]. Here, we first characterize the transient state through simulations and confront experimental signal profiles with simulated ones. We then compare the performance of multi-slice bSSFP to that of 3D bSSFP in human studies, and to that of single-slice bSSFP and multi-slice GE-EPI in rodent studies of BOLD fMRI.

\section{Materials and methods}

\subsection{Human brain at $7 \mathrm{~T}$}

\subsubsection{Simulations}

Bloch simulations were used to investigate the impact of prolonged relaxation between slab repetitions (number of slices: $1 / 2 / 4 / 8$ ) on the bSSFP magnetization profile. Simulations covered the frequency offset range $\left[-\frac{1}{\mathrm{TR}}, \frac{1}{\mathrm{TR}}\right]$ and flip angles between $5^{\circ}$ and $40^{\circ} . T_{1}$ and $T_{2}$ values were fixed at human gray matter values at $7 \mathrm{~T}$ : $2000 \mathrm{~ms}$ and $55 \mathrm{~ms}$, respectively [22,23]. The impact of realistic tissue modeling, diffusion and RF pulse duration on spin history $[24,25]$ was neglected. Simulations included the "catalyzation" (also called ramped preparation) module included with the product sequence on the scanner (TRUFI, Siemens VB17): a series of ten RF pulses with linear flip angle increase (e.g. $-\alpha / 4,+\alpha / 2,-3 \alpha / 4,+\alpha,-\alpha$, etc.), applied before each slice acquisition for rapid convergence towards pseudo-steady-state $[26,27]$ - Fig. 1 . The frequency range $\left[-\frac{1}{\mathrm{TR}}, \frac{1}{\mathrm{TR}}\right]$ was covered using incremental phase cycling steps [28], as implemented at the scanner. The bSSFP profile perturbations due to this phase cycling increase [29] were also simulated.

\subsubsection{Experimental}

The study was approved by the Committee for ethics in human research of the canton of Vaud (CER-VD, Switzerland). Experiments were performed on healthy volunteers using a $7 \mathrm{~T}$ head-only system (Siemens, Erlangen, Germany) and a 32-channel receiver coil (Nova Medical, Inc., MA, USA). Informed written consent was obtained from all participants.

Single- and multi-slice bSSFP signal profiles were acquired using: $\mathrm{TE} / \mathrm{TR}=2.9 / 5.8 \mathrm{~ms}$, matrix $52 \times 64$, in-plane resolution $3.5 \times 3.5 \mathrm{~mm}^{2}$, slice thickness $4.5 \mathrm{~mm}$, with $\mathrm{TR}_{\mathrm{vol}}=0.375 / 1.5 \mathrm{~s}$ and flip angle $15 / 30^{\circ}$ for one and four slices, respectively. The frequency range $\left[-\frac{1}{\mathrm{TR}}, \frac{1}{\mathrm{TR}}\right]$ was covered using incremental phase cycling steps [28] in order to minimize acquisition time. The influence of this fast acquisition protocol on the bSSFP profile was modeled using Bloch simulations, since this can result in a minor profile asymmetry, as detailed by Miller [29].

To compare single-slice and multi-slice functional contrast, multi-slice bSSFP resting-state fMRI (rs-fMRI) data were acquired
A

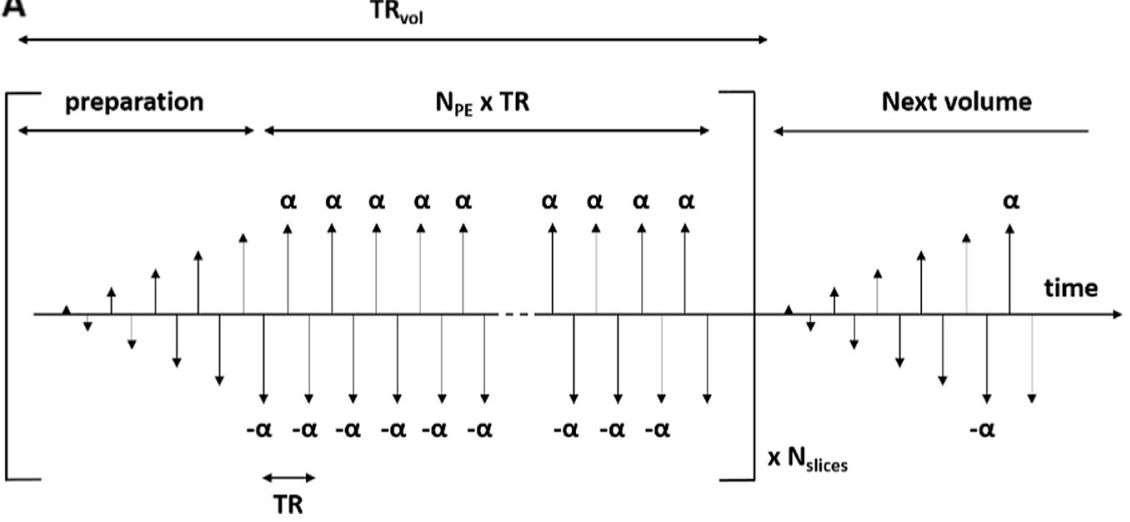

B

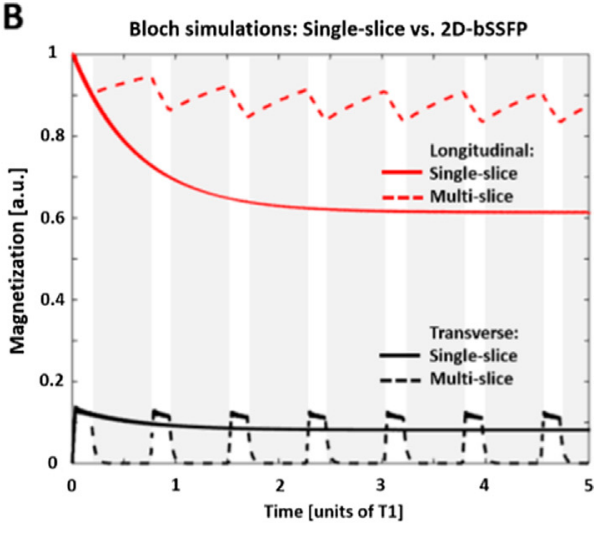

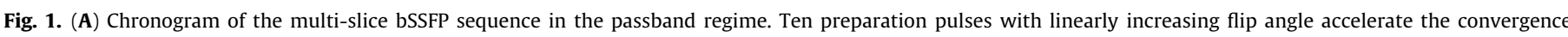

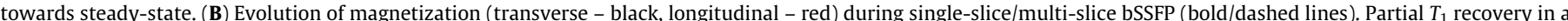

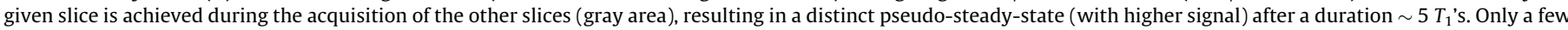

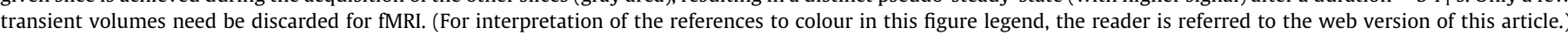


on 3 subjects ( $10 \mathrm{~min} /$ run) using the parameters above. Resting state networks (RSN) were extracted using the MELODIC toolbox of FSL [30] after brain extraction, smoothing (Gaussian kernel, FWHM $=6 \mathrm{~mm})$ and high-pass filtering $(f>0.01 \mathrm{~Hz})$. ICA decomposition was performed twice. In the first ICA decomposition, the number of dimensions was estimated automatically using the Laplace approximation to the Bayesian evidence of the model order as described in [31]. Artefactual components corresponding to physiological noise, motion or frequency fluctuations were manually sorted and removed from the data [32], and ICA was re-run on clean datasets. The number of ICA components in this second step was fixed to 30 as in [33]. The spatio-temporal patterns of the resulting RSNs were manually inspected and compared with a 10-RSN atlas [34].

Multi-slice (pseudo-steady-state) and 3D (steady-state) bSSFP images with matching parameters were acquired on six subjects: $\mathrm{TE} / \mathrm{TR}=2.51 / 5.02 \mathrm{~ms}$, matrix $=64 \times 60 \times 8$, in-plane/throughplane resolution $=3.5 / 4.5 \mathrm{~mm}$, the optimal flip angle was set to $40 / 15^{\circ}$ for $2 \mathrm{D} / 3 \mathrm{D}$ based on Bloch simulations using the $T_{1}$ and $T_{2}$ values of gray matter at $7 \mathrm{~T}, \mathrm{TR}_{\mathrm{vol}}=3000 / 2600 \mathrm{~ms}$, phase-cycling: $180^{\circ}$. $B_{1}$ maps were acquired prior to bSSFP acquisitions to ensure that the correct flip angle was reached. The SAR did not exceed $50 \%$ for any subject. The volume TR was longer for $2 \mathrm{D}$ due to the ramped preparation before each slice acquisition. 2D and 3D normalized temporal SNR maps $\left(\mathrm{tSNR}=\frac{\bar{s}_{t}}{\sigma_{t} \cdot \sqrt{T_{\mathrm{vol}}}}\right)$ were compared at whole-brain level (WB) and in gray matter (GM), white matter (WM) and cerebrospinal fluid (CSF) ROIs in mid-brain.

To compare multi-slice and 3D bSSFP fMRI performance, a finger-tapping task was run on three subjects. Each fMRI run consisted in blocks of $30 \mathrm{~s}$ of rest and $30 \mathrm{~s}$ of right-hand finger-tapping at $1 \mathrm{~Hz}$ frequency, repeated for five minutes. The data were analyzed using SPM12 (http://www.fil.ion.ucl.ac.uk/spm/software/ spm12/). The first six volumes were discarded to retain only multi-slice/3D bSSFP data in pseudo/true steady-state. The preprocessing pipeline included motion correction, slice timing correction and spatial smoothing (kernel FWHM equal to the voxel size). After general linear model (GLM) analysis using the canonical BOLD hemodynamic response function, the number of activated voxels at $p<0.05$ after family-wise error correction (FWE), and maximum/average T-scores amongst activated voxels $\left(\mathrm{T}_{\text {max }} / \mathrm{T}_{\text {mean }}\right)$ were compared.

\subsection{Rat brain at $14 \mathrm{~T}$}

\subsubsection{Animal preparation}

This study was approved by the Service for Veterinary Affairs of the canton of Vaud. Male Sprague-Dawley rats (Charles River, L'Arbresle, France) $(\mathrm{N}=13$; weight $=269 \pm 18 \mathrm{~g})$ were initially anesthetized with isoflurane ( $4 \%$ for induction and $2 \%$ for maintenance) and positioned in a homemade MRI cradle. Respiration rate and rectal temperature were monitored throughout the experiment (SA Instruments, Stony Brook, NY, USA). A catheter was inserted subcutaneously on the back of the animal for medetomidine delivery. For electrical stimulation during the fMRI experiment, two pairs of stainless steel electrodes were inserted in the forepaws, between digits 2 and 3 and digits 4 and 5 .

When the animal setup was finalized, the anesthesia was switched from isoflurane to medetomidine (Dorbene, Graeub, Switzerland): an initial bolus of $0.1 \mathrm{mg} / \mathrm{kg}$ was injected subcutaneously; the isoflurane was discontinued 10 min later (an oxygen/air supply of $20 \% / 80 \%$ was maintained throughout the experiment) and a continuous infusion of $0.1 \mathrm{mg} / \mathrm{kg} / \mathrm{h}$ medetomidine was started another five minutes later. The total sedation time under medetomidine was around three hours, with an average respiration rate of 85 breaths per minute. Medetomidine is free from the vasodilatory effects of isoflurane, which are deleterious both for BOLD sensitivity [35] and vein segmentation at ultrahigh field [36]. It should however be noted that medetomidine has vasoconstrictive effects which potentially also affect the BOLD signal. At the end of the experiment, animals were woken up with an intramuscular injection of atipamezole (Alzane, Graeub, Switzerland) at $0.5 \mathrm{mg} / \mathrm{kg}$ and returned to their cages.

\subsubsection{Multi-slice "pseudo steady-state" characterization}

Rat data were acquired on a 14-T Varian system (Abingdon, UK) equipped with $400 \mathrm{mT} / \mathrm{m}$ gradients. An in-house built quadrature surface coil was used for transmission and reception.

The aim of the multi-slice bSSFP was to match the brain coverage, spatial and temporal resolutions typically obtained with GEEPI on the same system: five $1-\mathrm{mm}$ thick coronal slices (interleaved acquisition), $300-400 \mu \mathrm{m}$ in-plane resolution, and $1.5 \mathrm{~s}$, respectively. Sequence parameters are given in Table 1.

Bloch simulations written in Matlab were used to generate theoretical signals for these multi-slice protocols, using measured relaxation times in the rat cortex at $14 \mathrm{~T}\left(T_{2}=25 \mathrm{~ms} /\right.$ $T_{1}=2200 \mathrm{~ms}$ ) and various flip angles. Properties of the transient signal in the multi-slice acquisition were compared to the steady-state signal.

Experimental single-slice and multi-slice bSSFP profiles were measured by varying the phase-cycling angle $\varphi$ from 0 to $360^{\circ}$ in steps of $30^{\circ}$, acquired in random order, with other imaging parameters listed in Table 1. The first few volumes were discarded until slab steady-state was established.

\subsubsection{Task-based fMRI}

For each fMRI run, either the left or the right paw was stimulated. The electrical stimulation consisted in square pulses of $0.3 \mathrm{~ms}$ at $2 \mathrm{~mA}$, with a frequency of $9 \mathrm{~Hz}$, delivered by an A365 stimulus isolator interfaced with a DS8000 digital stimulator (World Precision Instruments, Stevenage, UK), triggered by the MR scanner. Each fMRI run consisted in blocks of $21 \mathrm{~s}$ stimulation and $21 \mathrm{~s}$ rest, repeated for five minutes. Consecutive fMRI runs were separated by five minutes of full rest. The same paw was stimulated for two consecutive runs, before switching to the other paw.

The comparison between two types of protocols (i.e. multi-slice bSSFP vs single-slice bSSFP and multi-slice bSSFP vs GE-EPI) was performed using pairs of consecutive runs of stimulating the same paw, randomizing which protocol was acquired first, as detailed in Table 2.

All fMRI bSSFP images were acquired with a phase cycling of $180^{\circ}$ to center the main frequency on the passband. The bSSFP flip angle was adjusted for each protocol to optimize the extent and flatness of the passband plateau rather than the BOLD amplitude.

Table 1

Acquisition parameters for bSSFP and GE-EPI protocols.

\begin{tabular}{llll}
\hline & $\begin{array}{l}\text { Single-slice } \\
\text { bSSFP }\end{array}$ & $\begin{array}{l}\text { Multi-slice } \\
\text { bSSFP }\end{array}$ & $\begin{array}{l}\text { Multi-slice } \\
\text { GE-EPI }\end{array}$ \\
\hline TE/TR $(\mathrm{ms})$ & $3.064 / 6.128$ & & $17 / 1500$ \\
Number of slices & 1 & 5 & 5 \\
Dummy scans & 0 & 16 & 0 \\
Dummy volumes & 53 & 6 & 6 \\
TR $_{\text {vol }}(\mathrm{s})$ & 0.2 & 1.5 & 1.5 \\
Flip angle $\left({ }^{\circ}\right)$ & 12 & 22 & 62 \\
Bandwidth $(\mathrm{kHz})$ & 27 & & 250 \\
Matrix & $64 \times 32$ & & $64 \times 64$ \\
In-plane resolution $\left(\mu \mathrm{m}^{2}\right)$ & $300 \times 400$ & & $359 \times 359$ \\
Slice thickness $\left(\mathrm{mm}^{2}\right)$ & 1 & & 1 \\
Field of view $\left(\mathrm{mm}^{2}\right)$ & $19.2 \times 12.8$ & & $23 \times 23$ \\
\hline
\end{tabular}


Table 2

Number of rats involved in each comparison and total number of paired runs acquired for a given comparison.

\begin{tabular}{lll}
\hline Protocols compared & $\begin{array}{l}\text { Multi-slice vs single-slice } \\
\text { bSSFP }\end{array}$ & $\begin{array}{l}\text { GE-EPI vs multi-slice } \\
\text { bSSFP }\end{array}$ \\
\hline $\begin{array}{l}\text { No. of rats involved } \\
\text { Total pairs of runs }\end{array}$ & 7 & 7 \\
\hline
\end{tabular}

For anatomical reference, a high-resolution venogram was acquired using a 3D gradient echo sequence: $T E / T R=15 / 30 \mathrm{~ms} /$ flip angle: $20^{\circ}$ matrix: $256 \times 128 \times 128 / 100-\mu \mathrm{m}$ isotropic resolution/ $\mathrm{NA}=2 / \mathrm{TA}=16^{\prime}$ [37]

The data were analyzed using SPM12 (http://www.fil.ion.ucl.ac. $\mathrm{uk} / \mathrm{spm} / \mathrm{software} / \mathrm{spm} 12 /$ ). The processing pipeline included slice timing correction, spatial smoothing (kernel FWHM: 1.5 times the voxel size), and a square response function. The latter was deemed sufficient over an estimated rat hemodynamic response function because the paradigm used gave very robust activation. Statistical significance was retained at $p<0.05$ after FWE. Maximum $t$-score, cluster volume and BOLD signal amplitude were subsequently extracted. BOLD amplitude was estimated from the timecourses of the four voxels with highest $t$-score in the cluster. In a second step, when comparing single-slice to multi-slice bSSFP, the data with higher temporal resolution were downsampled to match the lower temporal resolution and reanalyzed to yield new $t$-scores and cluster volumes.

The SNR for each protocol and each run was estimated by performing principal component analysis on all temporal repetitions within a sliding $5 \times 5 \times 5$ voxel kernel and iteratively separating signal components from noise components, the latter being fit to a Marchenko-Pastur distribution [38]. In particular, the image SNR and temporal SNR - defined in the same way as for the human data - were compared between the multi-slice and single-slice bSSFP protocols.

Paired $t$-tests were run at the two-sided $5 \%$ significance level to determine significantly different metrics between protocols based on all available datasets (14 datasets for the multi-slice vs singleslice bSSFP comparison, 27 datasets for the multi-slice bSSFP vs GE-EPI comparison).

\section{Results}

\subsection{Human brain at $7 \mathrm{~T}$}

The evolution of the bSSFP transverse/longitudinal magnetization is illustrated in Fig. 1B. Compared to conventional (steadystate) bSSFP, higher flip angles were necessary to maximize the multi-slice bSSFP passband plateau width (Fig. 2), but the magnetization profiles obtained after flip angle-adjustment were similar. The multi-slice bSSFP pseudo-steady-state benefited from larger longitudinal magnetization recovery during the acquisition of neighboring slices. A slight bSSFP profile asymmetry was caused by the phase increment acquisition scheme (Fig. 3A, dotted versus plain lines). In vivo, the bSSFP profiles were found slightly asymmetric due to both the acquisition scheme and inhomogeneous intra-voxel frequency distributions [29,39]. Nevertheless, the simulations agreed extremely well with the experimental single-slice and multi-slice bSSFP signal profiles (Fig. 3B). Images confirmed the higher signal level of multi-slice bSSFP (associated with the transient state) (Fig. 3C).

The resting-state networks extracted from single-slice and multi-slice data were similar (in the matching slice), while multislice bSSFP benefitted from extended coverage and the identification of networks over a larger spatial scale (Fig. 4). Only the default mode, auditory, and two visual networks (occipital and lateral) were consistently detected on all subjects with multi-slice bSSFP data given the brain coverage available for this study. While visual and default mode networks could also be detected on all singleslice bSSFP data, the auditory network could only be detected in two out of three single-slice bSSFP datasets. A qualitative visual comparison of auditory, visual and default mode networks is provided for both sequences in a representative subject in the supplementary materials (Figure S1). A complete list of all 30 ICA components extracted from a representative single-slice bSSFP data is also available (Figure S2).

Temporal SNR was higher with multi-slice bSSFP than 3D-bSSFP in GM/WM/WB (Fig. 5A, $p<0.05$ ), due to a combination of higher signal in the pseudo steady-state and reduced sensitivity to motion. Indeed, motion had a reduced influence on multi-slice bSSFP signal than on its 3D counterpart (signal variance explained

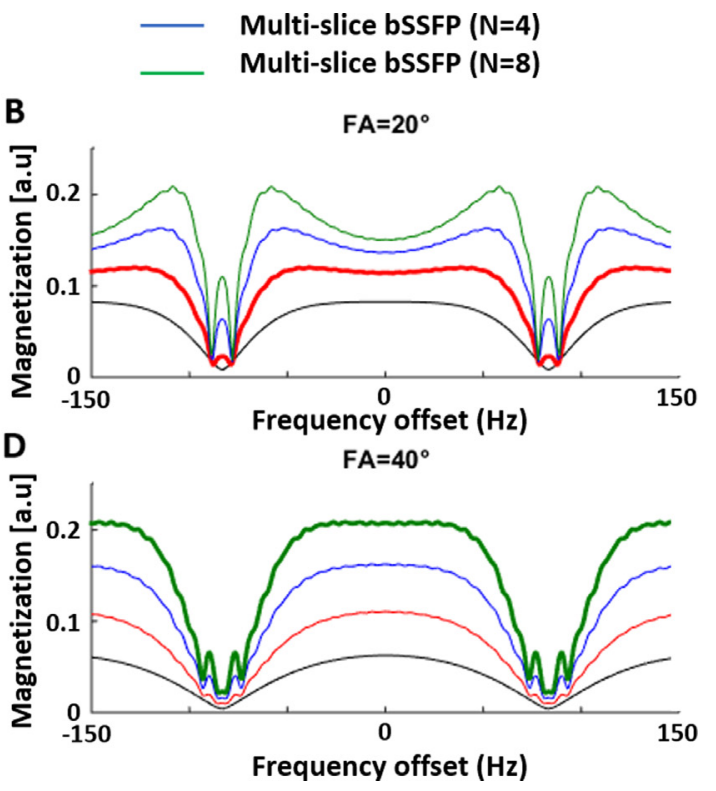

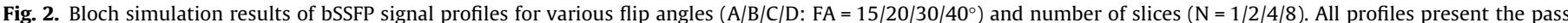

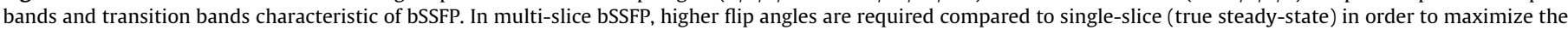
passband plateau. The optimal flip angle is highlighted in bold for $\mathrm{N}=1 / 2 / 4 / 8$. 
A. Bloch simulations

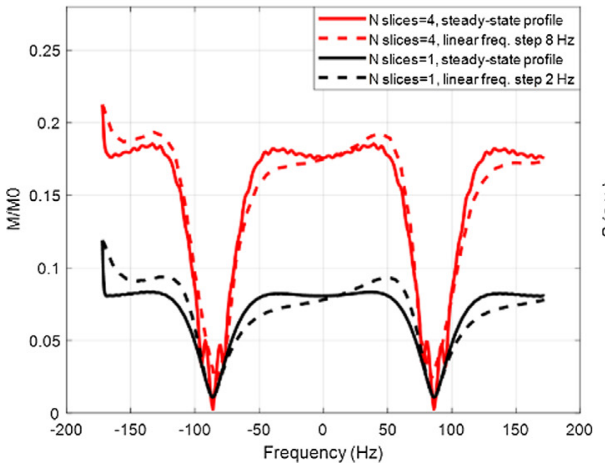

B. Single- / Multi-slice experimental profile

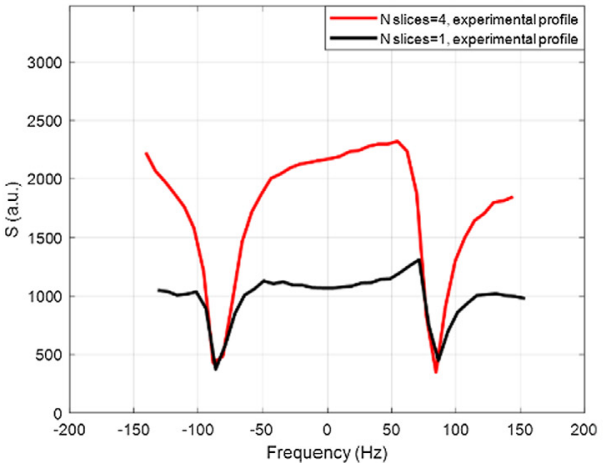

C. Single- / Multi-slice protocol image

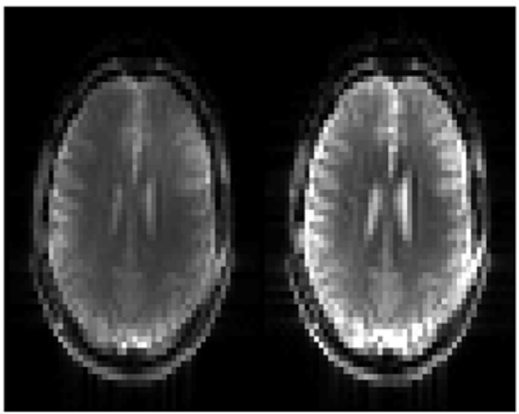

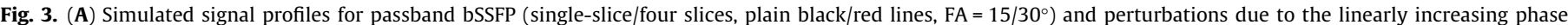

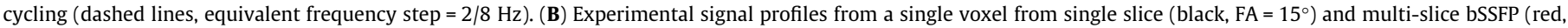

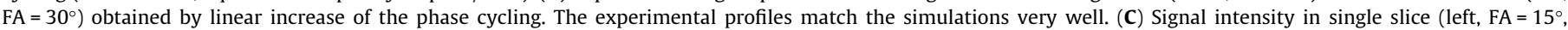

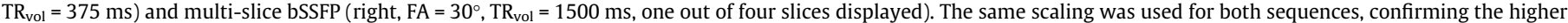
signal intensity for multi-slice bSSFP. (For interpretation of the references to colour in this figure legend, the reader is referred to the web version of this article.)

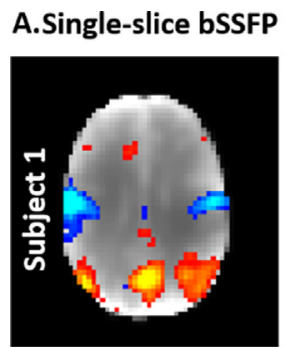

B.

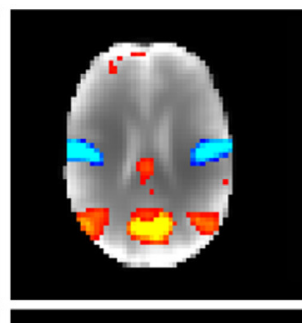

Multi-slice bSSFP (4 slices)
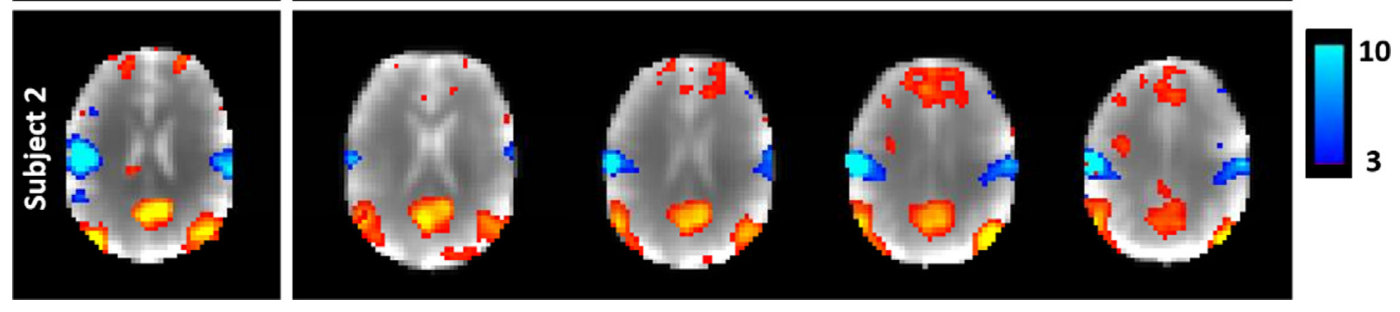

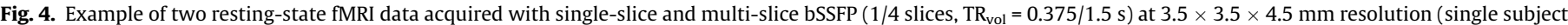

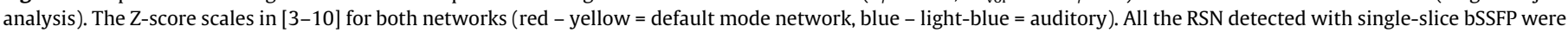

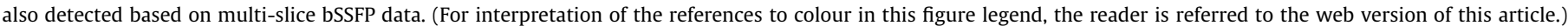

by motion $=7.5 / 16.4 \%$ for multi-slice/3D bSSFP, respectively, see Supplementary Figure S3). Furthermore, 3D-bSSFP suffered from aliasing along the second phase-encode direction because of imperfect slab selection, requiring the outer slices to be discarded (Fig. 5D). fMRI metrics (number of activated voxels, maximum and mean t-score) were similar between the two schemes (Fig. 5B). Both acquisition schemes detected a robust activation cluster in the motor cortex during finger-tapping, with no slices to be discarded in the multi-slice acquisition, as opposed to the 3D one which suffered from fold-over (Fig. 5C and D, representative subject).

\subsection{Rat brain at $14 \mathrm{~T}$}

The signal during multi-slice bSSFP acquisition at $14 \mathrm{~T}$ was simulated. While the signal did not reach steady-state during the acquisition of a given slice, the use of 16 dummy scans ensured that the transverse magnetization level was slowly varying (up to $14 \%$ ) such as not to introduce substantially uneven weighting in $k$-space (Fig. 6). The optimal flip angle for a passband profile in the steady-state was calculated to be $12^{\circ}\left(\cos \alpha=\frac{T_{1} / T_{2}-1}{T_{1} / T_{2}+1}\right)$ at $14 \mathrm{~T}$ [2]. In the multi-slice implementation, simulations showed the optimal flip angle to be dependent on sequence parameters (and essentially on the time available for longitudinal relaxation during the acquisition of other slices), with a value of $22^{\circ}$ for our protocol, which was also verified experimentally (Fig. 7A). A few initial volumes needed to be discarded before the slab steadystate was established (Fig. 7B).

We compared the multi-slice implementation to the single-slice acquisition at the same $\mathrm{TE}=3 \mathrm{~ms}$. Since it exploits the transient state, the SNR of the multi-slice protocol was higher than that of single-slice steady-state: $S N R=58 \pm 12$ for multi-slice and SNR $=38 \pm 7$ for single-slice in the somatosensory cortex, as also predicted by simulations (Fig. 7A). The temporal SNR for the single-slice protocol was naturally higher $\left(\mathrm{tSNR}_{\mathrm{ss}}=85 \mathrm{~s}^{-1 / 2}\right.$ vs $\mathrm{tSNR}_{\mathrm{ms}}=47 \mathrm{~s}^{-1 / 2}$ ) but the difference in spatial coverage between protocols still speaks in favor of multi-slice bSSFP. Indeed, although lower, the temporal SNR of the multi-slice protocol was nonetheless sufficient to robustly detect BOLD response (see Fig. 8), in which case it becomes preferable to have increased brain coverage at the expense of lower temporal resolution, in order to detect activation that potentially spans more than one slice.

In terms of task-fMRI metrics, both cluster size $(p=4.27 \mathrm{E}-04)$ and $t$-score $(p=8.91 \mathrm{E}-05)$ were higher with the single-slice sequence vs multi-slice (due to higher tSNR for single-slice), 


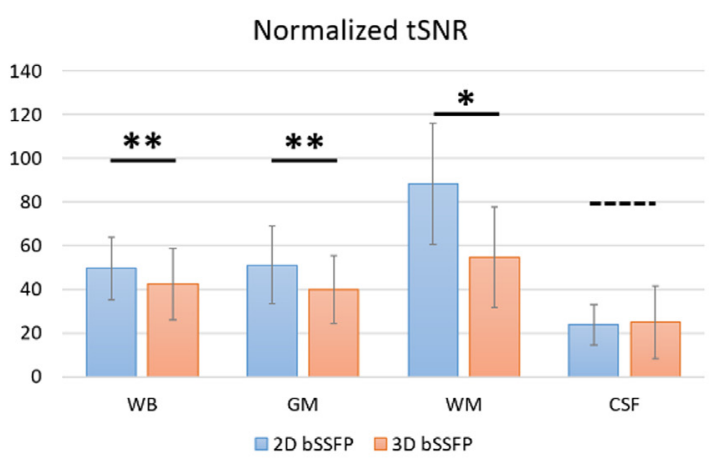

B

\begin{tabular}{|c|cc|cc|cc|}
\hline Subject & \multicolumn{2}{c}{ N $_{\text {FWE }}$} & \multicolumn{2}{c}{$T_{\text {max }}$} & \multicolumn{2}{c|}{$T_{\text {mean }}$} \\
\hline & 2D & 3D & 2D & 3D & 2D & 3D \\
$\# 1$ & 273 & 234 & 14.0 & 16.6 & 7.2 & 7.3 \\
$\# 2$ & 112 & 48 & 16.0 & 13.7 & 7.5 & 6.5 \\
$\# 3$ & 46 & 59 & 12.5 & 9.8 & 6.8 & 6.1 \\
Mean (std) & $144(117)$ & $114(104)$ & $14.2(1.7)$ & $13.4(3.4)$ & $7.2(0.3)$ & $6.6(0.6)$ \\
\hline
\end{tabular}

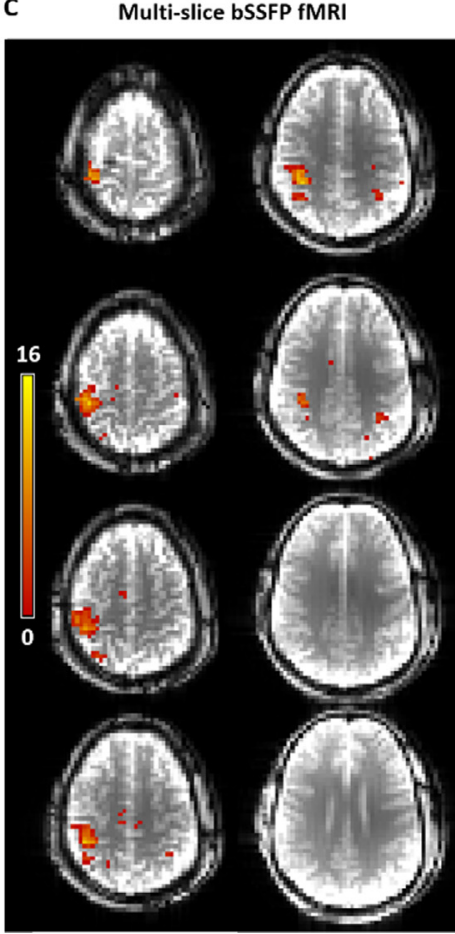

D

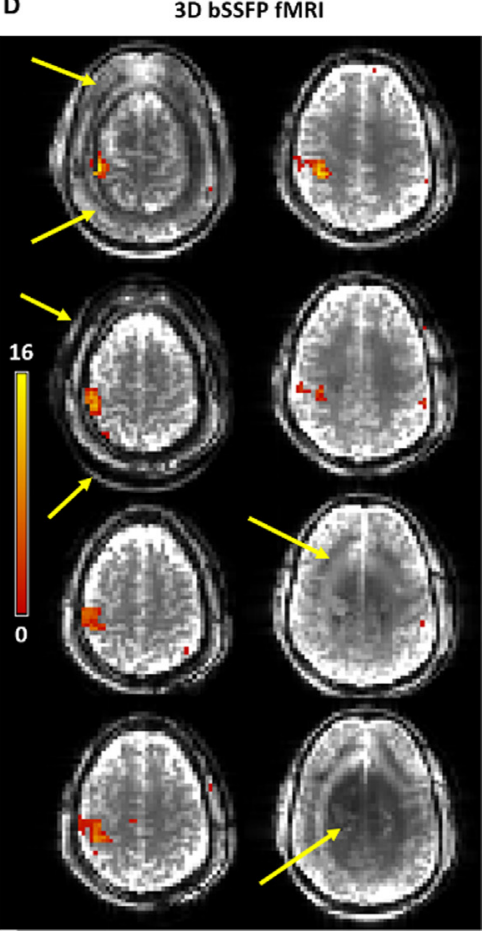

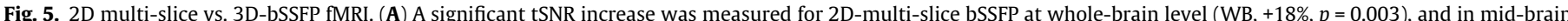

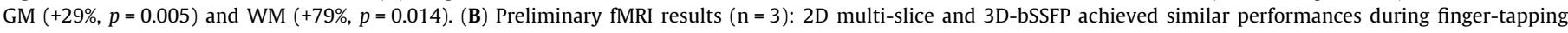

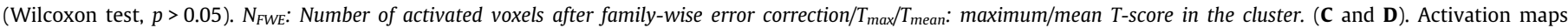

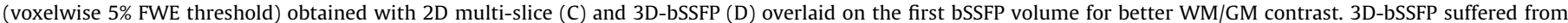

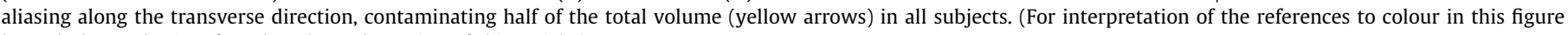
legend, the reader is referred to the web version of this article.)

though after down-sampling the single-slice to the same temporal resolution, cluster size became similar and $t$-score significantly lower ( $p=1.35 \mathrm{E}-04$ ) compared to multi-slice bSSFP (due to the higher image SNR in the latter). The BOLD amplitude was other-

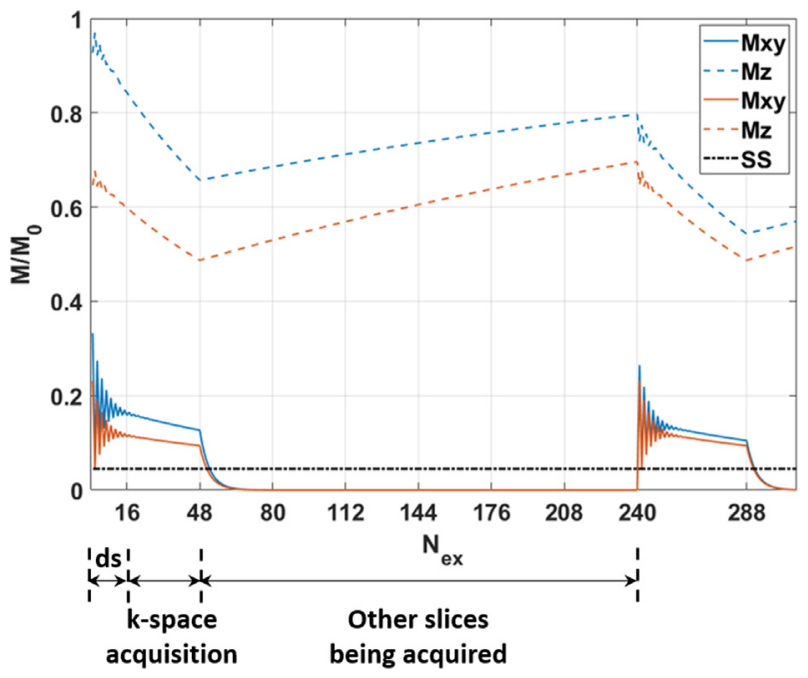

Fig. 6. Simulated bSSFP signal evolution during the acquisition of five slices, with 16 dummy scans (ds) and 32 phase encoding steps, for $\mathrm{TE} / \mathrm{TR}=3 / 6 \mathrm{~ms}$. The blue curves represent the first two consecutive repetitions in a run, where the signal level still varies between repetitions, and the red curves represent two later consecutive repetitions, when slab steady-state is established. Each k-space acquisition still occurs in the transient phase of bSSFP, but the transverse magnetization Mxy is slowly varying. The true steady-state (SS) level for Mxy is shown in black. (For interpretation of the references to colour in this figure legend, the reader is referred to the web version of this article.) wise comparable between the two protocols $(1.4 \pm 0.4 \%$ and $1.6 \pm 0.5 \%$, respectively) (Fig. 8).

Activation clusters were very similar between GE-EPI and multi-slice bSSFP (Fig. 9A), with bSSFP images additionally more readily registrable to an anatomical reference (Supplementary Figure S4). While the BOLD amplitude was significantly higher with GE-EPI than bSSFP ( $p=4.03 \mathrm{E}-04)$, cluster size and maximum $t$-score were comparable between the two sequences (Fig. 9B-C).

\section{Discussion and conclusions}

This study demonstrates that multi-slice bSSFP is suitable for resting-state and task-fMRI on a thin slab, and provides distortion-free activation maps at high and ultra-high field. A straightforward multi-slice bSSFP implementation with a $180^{\circ}$ phase cycling was used, which is likely available on any MR system.

The multi-slice acquisition breaks the steady-state and $k$-space data is acquired in a transient, albeit slowly varying state. The transient state was characterized both through simulations and experimental data, and exhibited similar properties to the steady-state for passband BOLD fMRI purposes, while benefitting from higher signal. The flip angle was increased relative to the optimal steady-state flip angle to retrieve a maximally flat passband. It should be noted that the $T_{2}$ and $T_{1}$ weighting of the transient signal are different from that of the steady-state [21] which can be expected to impact the BOLD contrast. However, using tasks which reputedly produce robust responses, very similar BOLD amplitude and activation maps were obtained between single-slice and multi-slice bSSFP in both human brain (Fig. 4) and rat brain (Fig. 8). It should be noted that signal differences between steady-state and pseudo-steady-state could potentially play a 


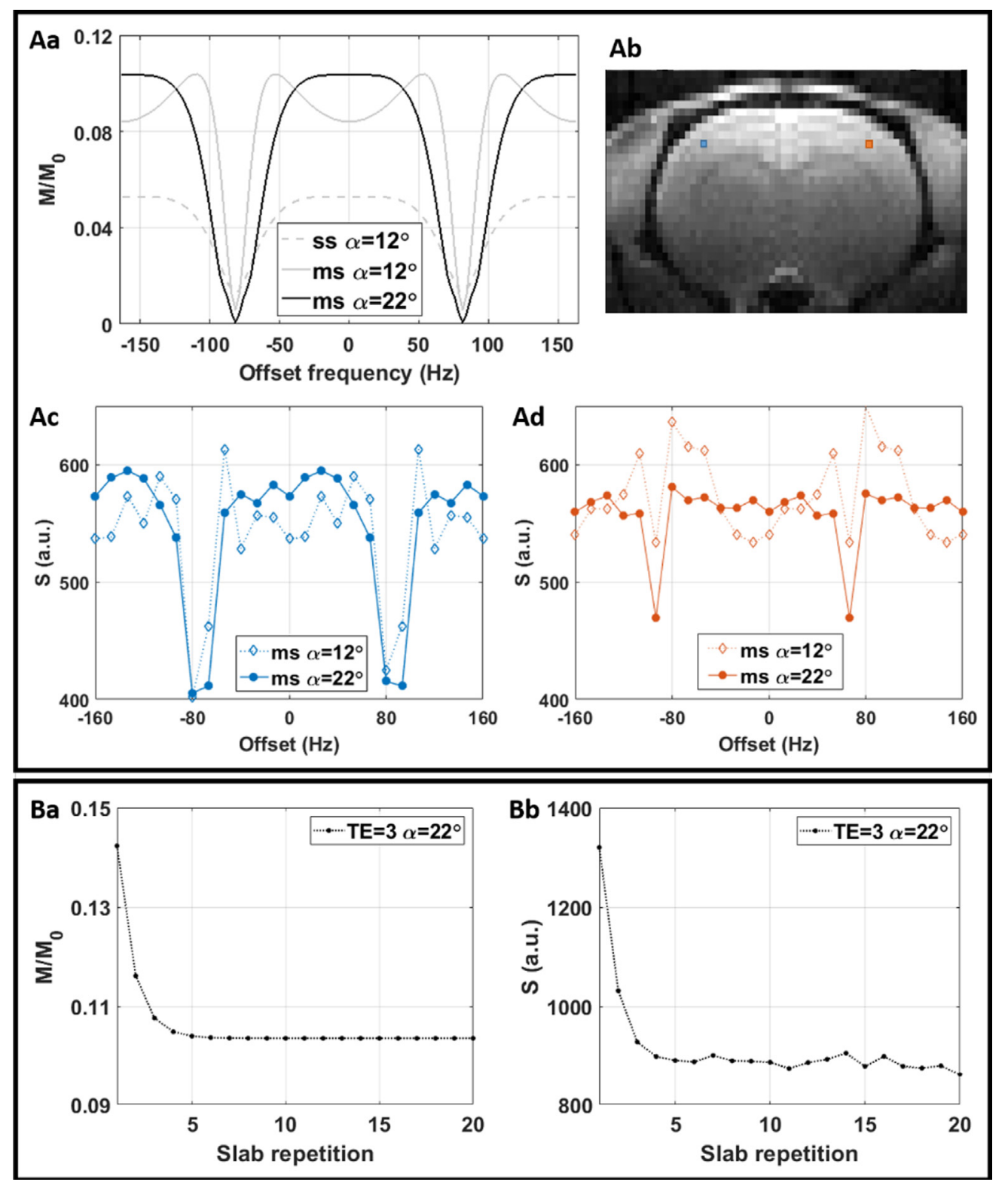

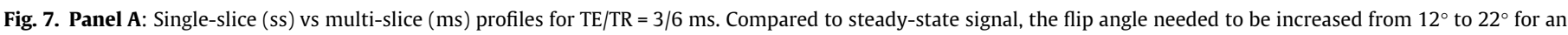

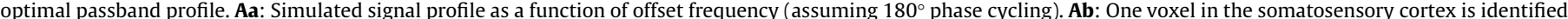

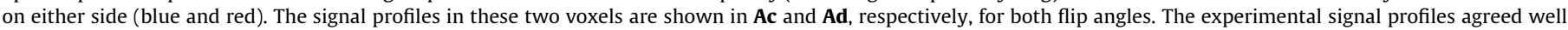

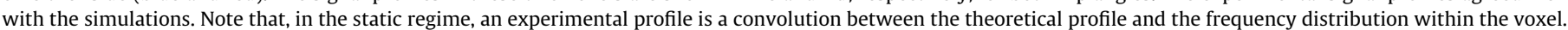

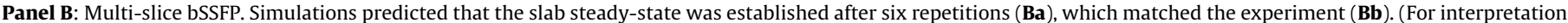
of the references to colour in this figure legend, the reader is referred to the web version of this article.)

more significant role in study designs investigating more subtle BOLD effects than the tasks used here. Although the number of subjects can be considered low compared to a conventional neuroscience/cognitive fMRI study, it is well sufficient to appreciate the performance of each type of sequence examined. A larger number of subjects would be needed to examine potential quantitative differences in functional connectivity or task-response related to the use of one or the other sequence, which is beyond the scope of this study.

On the human $7 \mathrm{~T}$ system, costs in temporal resolution due to the need to re-establish steady-state between slices (compared to 3D bSSFP) were minimized by the use of catalyzation [26] to accelerate convergence towards steady-state, and mitigated by higher signal in the new steady-state. Multi-slice bSSFP tSNR values were higher than $3 \mathrm{D}$, also as a byproduct of reduced motion/ physiological noise. This effect was already shown for unaccelerated multi-slice and 3D-GE-EPI [40], resulting in superior or equal performances for task-fMRI, in addition to reduced image artefacts. At high field, many regions affected by strong magnetic susceptibility mismatch and that benefit from non-EPI acquisitions - such as the brainstem and retina - are unfortunately also subject to major flow or motion artefacts originating from the blood vessels or the eyes, making 3D-bSSFP acquisitions problematic as well. In that regard, motion-related artifacts are substantially reduced when using a multi-slice approach, and multi-slice bSSFP might be better suited than its 3D counterpart for distortion-free localized fMRI applications, such as retina fMRI [13].

In the absence of acceleration, spatial resolution was kept low in order to adequately probe the BOLD hemodynamic response function during fMRI. Although this approach was sufficient for 

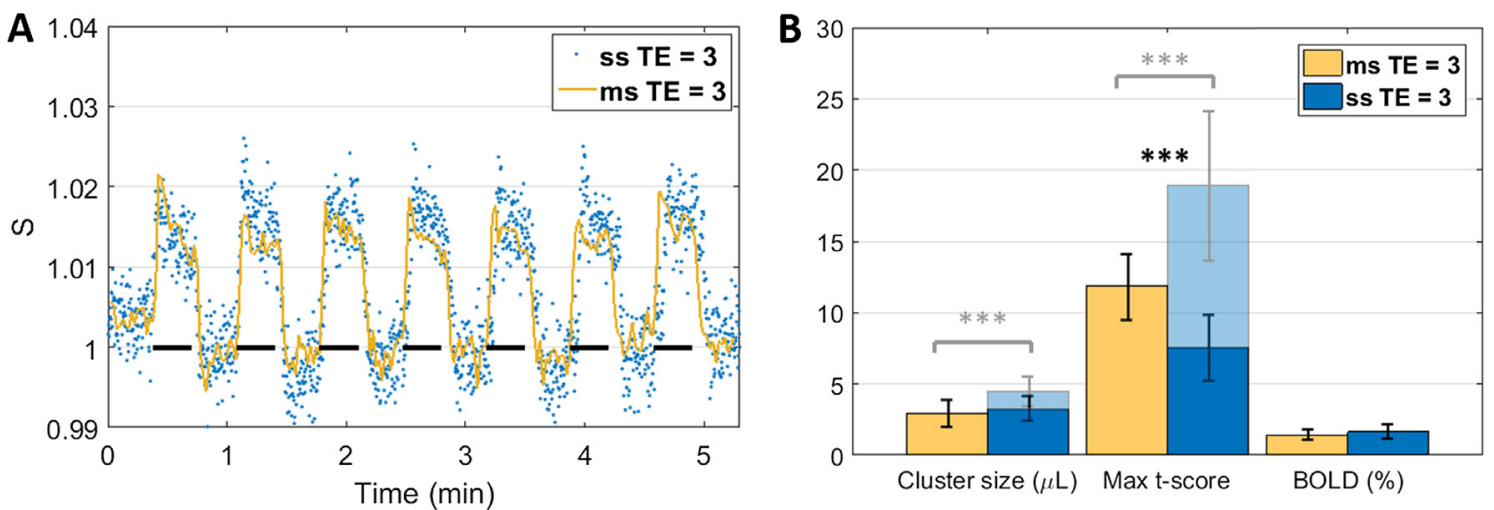

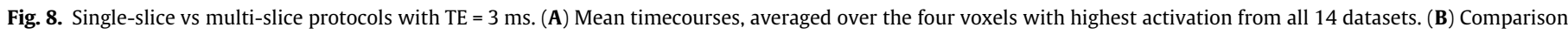

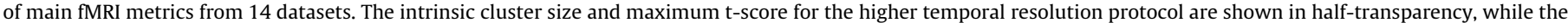
recalculated values at matched temporal resolutions are shown in solid.

A
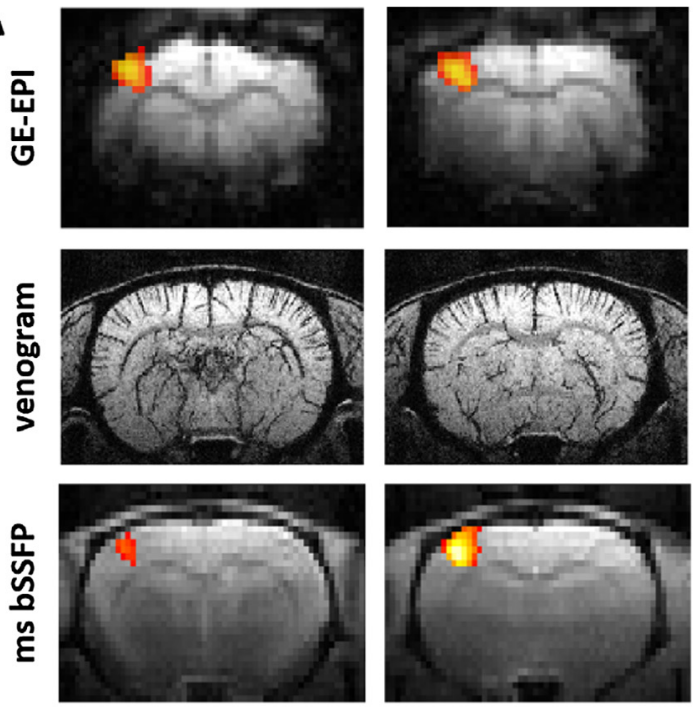

B

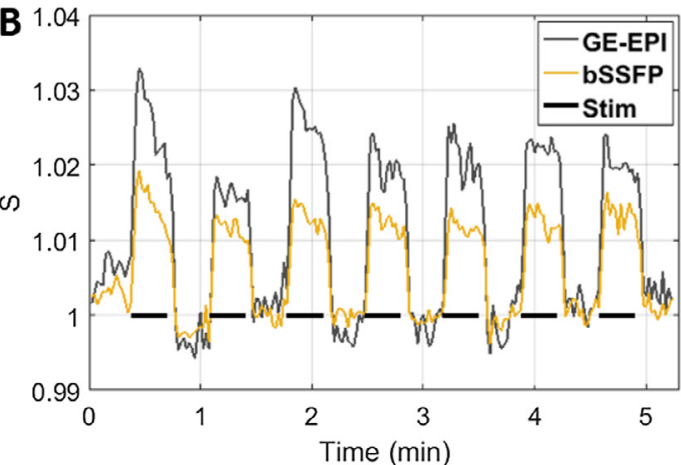

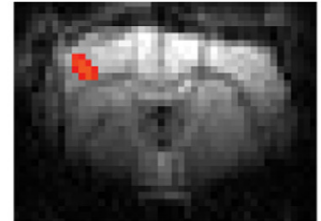
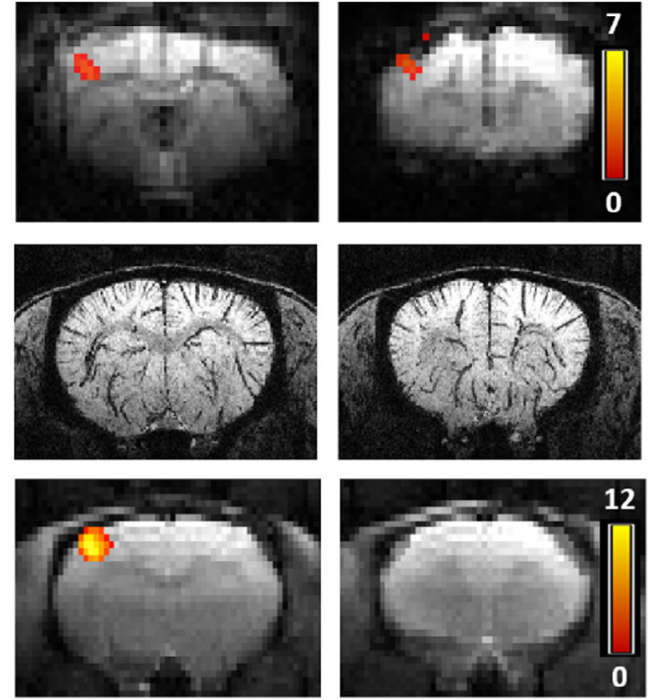

$C^{1}$

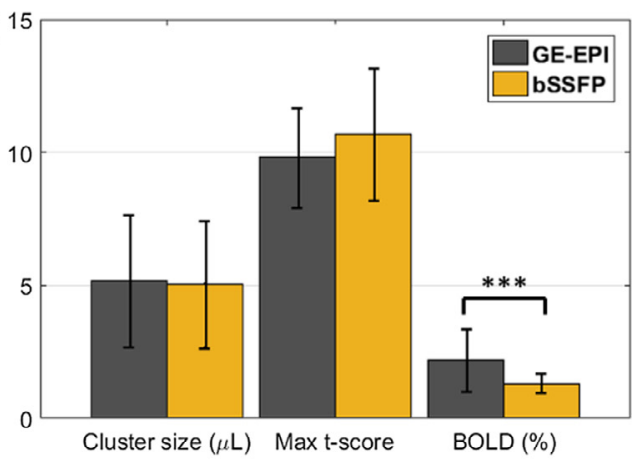

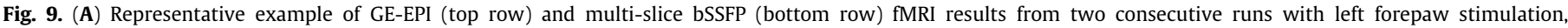

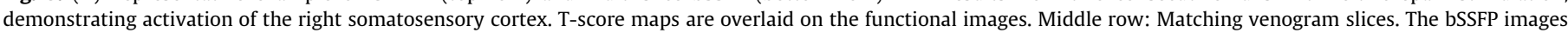

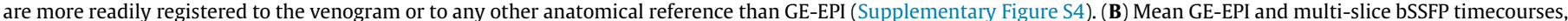

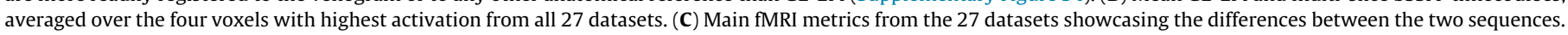

this proof-of-concept study, a practical usage of multi-slice bSSFP for task or resting-state fMRI with higher spatial resolution would indeed require the implementation of acceleration capabilities. Accelerated 3D-bSSFP has already shown great potential for distortion-free imaging at high field applied to fMRI [18-20,41] or Arterial Spin Labeling [42]. Future work could focus on combining multi-slice bSSFP with in-slice (GRAPPA) and through-slice
(SMS) acceleration, as a less motion-sensitive alternative to accelerated 3D-bSSFP with similar brain coverage and/or spatial resolution. Alternatively, the pseudo steady-state generated by alternating-SSFP with accelerated 3D readout can be optimized as described in this study to generate rapid distortion-free and artefact-free 3D-bSSFP functional contrast at $7 \mathrm{~T}$ [43], similarly to a previous implementation at $3 \mathrm{~T}$ [44]. 
On the rodent $14 \mathrm{~T}$ system, the multi-slice acquisition represents a compromise between brain coverage (which needs to extend beyond the single slice for proper coverage of a given functional area) and temporal resolution, which would likely be too low in a full Cartesian 3D acquisition. While the availability of multichannel receive arrays for rodents remains limited, so do acceleration options. One group reported using stacks of spirals for 3D bSSFP fMRI in rats [45], while another implemented compressed sensing for single-slice bSSFP fMRI [12]. These acceleration options could in principle be combined with a multi-slice bSSFP acquisition, and a formal comparison between multi-slice and 3D bSSFP fMRI could be performed, but the human data in this work suggest the benefit of multi-slice bSSFP would be maintained. The preclinical implementation of the sequence would also benefit from a ramped preparation scheme to reduce the number of dummy scans required to reach the transient state signal - currently one third of the time was spent on dummy scans. Alternatively, a center-in encoding could be used with fewer dummy scans to produce the same quasi-steady-state for the central k-space line [44], but such schemes are more sensitive to eddy currents.

Remarkably, bSSFP provided similar activation maps to GE-EPI in the rodent brain. In spite of a lower BOLD amplitude for bSSFP, related to the shorter TE than that of GE-EPI and the different contrast mechanisms, activation epochs were detected very reliably, achieving similar cluster size and $t$-statistics. We underline that our goal was to perform a practical comparison between the two sequences by matching their temporal and spatial resolutions, as well as brain coverage, rather than a formal comparison of BOLD contrast mechanisms between bSSFP and GE (e.g. by matching their TE's) as investigated by other groups $[5,6,9,11]$. Previous practical comparisons of bSSFP to GE-EPI performed at $7 \mathrm{~T}$ in rats $[13,14]$, indicating similar activation detection for both task and resting-state fMRI, seem to extend to $14 \mathrm{~T}$. It should be noted that bSSFP images are also less prone to distortions by field inhomogeneity, very prominent at ultra-high field, and are more easily registered to an anatomical reference than GE-EPI. The multislice bSSFP acquisition is expected to be increasingly beneficial for mouse brain [16]: the smaller skull size of mice leads to larger distortions in GE-EPI images, but is not expected to affect bSSFP images in a major way.

Compared to 3D bSSFP or multi-slice GE-EPI, multi-slice bSSFP in a pseudo-steady-state therefore combines substantial advantages in terms of motion insensitivity, spatial coverage and image quality for human and rodent fMRI at high field, with no penalty in terms of BOLD sensitivity. Such a protocol can be readily implemented on any MR system and thus constitutes a valuable approach for fMRI, particularly in regions notoriously challenging at high field such as retina, brainstem or temporal/occipital lobes.

\section{Acknowledgments}

The authors thank Klaus Scheffler and Valerij Kiselev for insightful discussions. They also acknowledge assistance with animal setup and monitoring from Mario Lepore and Stefan Mitrea. This work was supported by the Centre d'Imagerie Bio-Médicale (CIBM) of the University of Lausanne (UNIL), the Swiss Federal Institute of Technology Lausanne (EPFL), the University of Geneva (UniGe), the Centre Hospitalier Universitaire Vaudois (CHUV), the Hôpitaux Universitaires de Genève (HUG) and the Leenaards and the Jeantet Foundations.

\section{Appendix A. Supplementary material}

Supplementary data to this article can be found online at https://doi.org/10.1016/j.jmr.2019.05.010.

\section{References}

[1] C.V. Bowen, R.S. Menon, J.S. Gati, High field balanced-SSFP FMRI: a BOLD technique with excellent tissue sensitivity and superior large vessel suppression, in: Proc. of the ISMRM, Miami, FL, USA, 2005, pp. 119.

[2] K. Scheffler, S. Lehnhardt, Principles and applications of balanced SSFP techniques, Eur. Radiol. 13 (2003) 2409-2418.

[3] K.L. Miller, B.A. Hargreaves, J. Lee, D. Ress, R.C. deCharms, J.M. Pauly, Functional brain imaging using a blood oxygenation sensitive steady state, Magnetic Resonance Med.: Off. J. Soc. Magnet. Reson. Med./Soc. Magnet. Reson. Med. 50 (2003) 675-683.

[4] K. Scheffler, E. Seifritz, D. Bilecen, R. Venkatesan, J. Hennig, M. Deimling, E.M. Haacke, Detection of BOLD changes by means of a frequency-sensitive trueFISP technique: preliminary results, NMR Biomed. 14 (2001) 490-496.

[5] K.L. Miller, S.M. Smith, P. Jezzard, G.C. Wiggins, C.J. Wiggins, Signal and noise characteristics of SSFP FMRI: a comparison with GRE at multiple field strengths, NeuroImage 37 (2007) 1227-1236.

[6] S.H. Park, T. Kim, P. Wang, S.G. Kim, Sensitivity and specificity of highresolution balanced steady-state free precession fMRI at high field of 9.4T, NeuroImage, 58 (2011) 168-176.

[7] K.L. Miller, FMRI using balanced steady-state free precession (SSFP), NeuroImage 62 (2012) 713-719.

[8] T.S. Kim, J. Lee, J.H. Lee, G.H. Glover, J.M. Pauly, Analysis of the BOLD characteristics in pass-band bSSFP fMRI, Int. J. Imaging Syst. Technol. 22 (2012) $23-32$.

[9] K. Zhong, J. Leupold, J. Hennig, O. Speck, Systematic investigation of balanced steady-state free precession for functional MRI in the human visual cortex at 3 Tesla, Magnet. Reson. Med.: Off. J. Soc. Magnet. Resonan. Med./Soc. Magnet. Reson. Med. 57 (2007) 67-73.

[10] K.L. Miller, P. Jezzard, Modeling SSFP functional MRI contrast in the brain, Magnet. Reson. Med.: Off. J. Soc. Magnet. Reson. Med./Soc. Magnet. Reson. Med. 60 (2008) 661-673.

[11] M.G. Baez-Yanez, P. Ehses, C. Mirkes, P.S. Tsai, D. Kleinfeld, K. Scheffler, The impact of vessel size, orientation and intravascular contribution on the neurovascular fingerprint of BOLD bSSFP fMRI, NeuroImage 163 (2017) 13-23.

[12] P.K. Han, S.H. Park, S.G. Kim, J.C. Ye, Compressed sensing for fMRI: feasibility study on the acceleration of non-EPI fMRI at 9.4T, BioMed Research International 2015 (2015) 131926.

[13] E.R. Muir, T.Q. Duong, Layer-specific functional and anatomical MRI of the retina with passband balanced SSFP, Magnet. Reson. Med.: Off. J. Soc. Magnet. Reson. Med./Soc. Magnet. Reson. Med. 66 (2011) 1416-1421.

[14] J.S. Cheng, P.P. Gao, I.Y. Zhou, R.W. Chan, Q. Chan, H.K. Mak, P.L. Khong, E.X. $\mathrm{Wu}$, Resting-state fMRI using passband balanced steady-state free precession, PloS one 9 (2014) e91075.

[15] I.Y. Zhou, M.M. Cheung, C. Lau, K.C. Chan, E.X. Wu, Balanced steady-state free precession fMRI with intravascular susceptibility contrast agent, Magnet. Reson. Med.: Off. J. Soc. Magnet. Reson. Med./Soc. Magnet. Reson. Med. 68 (2012) 65-73.

[16] G. Blazquez Freches, C. Chavarrias, N. Shemesh, BOLD-fMRI in the mouse auditory pathway, NeuroImage 165 (2018) 265-277.

[17] T. Benkert, P. Ehses, M. Blaimer, P.M. Jakob, F.A. Breuer, Fast isotropic bandingfree bSSFP imaging using 3D dynamically phase-cycled radial bSSFP (3D DYPRSSFP), Zeitschrift fur medizinische Physik 26 (2016) 63-74.

[18] J.H. Lee, S.O. Dumoulin, E.U. Saritas, G.H. Glover, B.A. Wandell, D.G. Nishimura, J.M. Pauly, Full-brain coverage and high-resolution imaging capabilities of passband b-SSFP fMRI at 3T, Magn. Reson. Med. 59 (2008) 1099-1110.

[19] P. Ehses, K. Scheffler, Multiline balanced SSFP for rapid functional imaging at ultrahigh field, Magnet. Reson. Med.: Off. J. Soc. Magnet. Reson. Med./Soc. Magnet. Reson. Med. 79 (2018) 994-1000.

[20] M. Chappell, A.K. Haberg, A. Kristoffersen, Balanced steady-state free precession with parallel imaging gives distortion-free fMRI with high temporal resolution, Magn. Reson. Imaging 29 (2011) 1-8.

[21] K. Scheffler, On the transient phase of balanced SSFP sequences, Magnet. Reson. Med.: Off. J. Soc. Magnet. Reson. Med./Soc. Magnet. Reson. Med. 49 (2003) 781-783.

[22] P.J. Wright, O.E. Mougin, J.J. Totman, A.M. Peters, M.J. Brookes, R. Coxon, P.E Morris, M. Clemence, S.T. Francis, R.W. Bowtell, P.A. Gowland, Water proton T1 measurements in brain tissue at 7, 3, and 1.5 T using IR-EPI, IR-TSE, and MPRAGE: results and optimization, Magma (New York, N.Y.) 21 (2008) 121130.

[23] E. Yacoub, T.Q. Duong, P.F. Van De Moortele, M. Lindquist, G. Adriany, S.G. Kim, K. Ugurbil, X. Hu, Spin-echo fMRI in humans using high spatial resolutions and high magnetic fields, Magnet. Reson. Med.: Off. J. Soc. Magnet. Reson. Med./ Soc. Magnet. Reson. Med. 49 (2003) 655-664.

[24] O. Bieri, K. Scheffler, Effect of diffusion in inhomogeneous magnetic fields on balanced steady-state free precession, NMR Biomed. 20 (2007) 1-10.

[25] O. Bieri, K. Scheffler, SSFP signal with finite RF pulses, Magnet. Reson. Med.: Off. J. Soc. Magnet. Reson. Med./Soc. Magnet. Reson. Med. 62 (2009) 1232 1241.

[26] V.S. Deshpande, Y.C. Chung, Q. Zhang, S.M. Shea, D. Li, Reduction of transient signal oscillations in true-FISP using a linear flip angle series magnetization preparation, Magnet. Reson. Med.: Off. J. Soc. Magnet. Reson. Med./Soc. Magnet. Reson. Med. 49 (2003) 151-157.

[27] P. Le Roux, Simplified model and stabilization of SSFP sequences, J. Magn. Reson. 163 (2003) 23-37. 
[28] S. Patterson, S.D. Beyea, C.V. Bowen, FMRI using high flip-angle alternating steady state balanced SSFP supported by Monte Carlo studies, in: Proc. Intl. Soc. Magn. Reson. Med. 19, Montreal, Canada, 2011, pp. 1630.

[29] K.L. Miller, Asymmetries of the balanced SSFP profile Part I: theory and observation, Magnet. Reson. Med.: Off. J. Soc. Magnet. Reson. Med./Soc. Magnet. Reson. Med. 63 (2010) 385-395.

[30] S.M. Smith, M. Jenkinson, M.W. Woolrich, C.F. Beckmann, T.E. Behrens, H. Johansen-Berg, P.R. Bannister, M. De Luca, I. Drobnjak, D.E. Flitney, R.K. Niazy, J. Saunders, J. Vickers, Y. Zhang, N. De Stefano, J.M. Brady, P.M. Matthews, Advances in functional and structural MR image analysis and implementation as FSL, Neurolmage 23 (Suppl 1) (2004) S208-S219.

[31] C.F. Beckmann, S.M. Smith, Probabilistic independent component analysis for functional magnetic resonance imaging, IEEE Trans. Med. Imaging 23 (2004) $137-152$.

[32] L. Griffanti, G. Douaud, J. Bijsterbosch, S. Evangelisti, F. Alfaro-Almagro, M.F. Glasser, E.P. Duff, S. Fitzgibbon, R. Westphal, D. Carone, C.F. Beckmann, S.M. Smith, Hand classification of fMRI ICA noise components, Neurolmage 154 (2017) 188-205.

[33] S.M. Smith, C.F. Beckmann, J. Andersson, E.J. Auerbach, J. Bijsterbosch, G. Douaud, E. Duff, D.A. Feinberg, L. Griffanti, M.P. Harms, M. Kelly, T. Laumann, K. L. Miller, S. Moeller, S. Petersen, J. Power, G. Salimi-Khorshidi, A.Z. Snyder, A.T. Vu, M.W. Woolrich, J. Xu, E. Yacoub, K. Uğurbil, D.C. Van Essen, M.F. Glasser, Resting-state fMRI in the human connectome project, Neurolmage 80 (2013) $144-168$.

[34] S.M. Smith, P.T. Fox, K.L. Miller, D.C. Glahn, P.M. Fox, C.E. Mackay, N. Filippini, K.E. Watkins, R. Toro, A.R. Laird, C.F. Beckmann, Correspondence of the brain's functional architecture during activation and rest, PNAS 106 (2009) 1304013045.

[35] H. Takuwa, T. Matsuura, T. Obata, H. Kawaguchi, I. Kanno, H. Ito, Hemodynamic changes during somatosensory stimulation in awake and isofluraneanesthetized mice measured by laser-Doppler flowmetry, Brain Res. 1472 (2012) 107-112.
[36] L. Ciobanu, O. Reynaud, L. Uhrig, B. Jarraya, D. Le Bihan, Effects of anesthetic agents on brain blood oxygenation level revealed with ultra-high field MRI, PloS one 7 (2012) e32645.

[37] S.-H. Park, K. Masamoto, K. Hendrich, I. Kanno, S.-G. Kim, Imaging brain vasculature with BOLD 3D microscopy: MR detection limits determined by in vivo two-photon microscopy, Magnet. Reson. Med.: Off. J. Soc. Magnet. Reson. Med./Soc. Magnet. Reson. Med. 59 (2008) 855-865.

[38] J. Veraart, E. Fieremans, D.S. Novikov, Diffusion MRI noise mapping using random matrix theory, Magn. Reson. Med. (2015) n/a-n/a.

[39] K.L. Miller, S.M. Smith, P. Jezzard, Asymmetries of the balanced SSFP profile Part II: white matter, Magnet. Reson. Med.: Off. J. Soc. Magnet. Reson. Med./ Soc. Magnet. Reson. Med. 63 (2010) 396-406.

[40] O. Reynaud, J. Jorge, R. Gruetter, J.P. Marques, W. van der Zwaag, Influence of physiological noise on accelerated 2D and 3D resting state functional MRI data at 7 T., Magnet. Reson. Med.: Off. J. Soc. Magnet. Reson. Med./Soc. Magnet. Reson. Med. 78 (2017) 888-896.

[41] K. Scheffler, P. Ehses, High-resolution mapping of neuronal activation with balanced SSFP at 9.4 tesla, Magnet. Reson. Med.: Off. J. Soc. Magnetic Reson. Med. Soc. Magnet. Reson. Med. 76 (2016) 163-171.

[42] P.K. Han, J.C. Ye, E.Y. Kim, S.H. Choi, S.H. Park, Whole-brain perfusion imaging with balanced steady-state free precession arterial spin labeling, NMR Biomed. 29 (2016) 264-274.

[43] O. Reynaud, I. Jelescu, R. Gruetter, Band-free Whole-brain Alternating SSFP fMRI Provides Distortion-free Activation Maps in the Visual Cortex at 7T, in: ISMRM, France, Paris, 2018, p. 711.

[44] T. Jou, S. Patterson, J.M. Pauly, C.V. Bowen, Fat-suppressed alternating-SSFP for whole-brain fMRI using breath-hold and visual stimulus paradigms, Magnet. Reson. Med.: Off. J. Soc. Magnet. Reson. Med. Soc. Magnet. Reson. Med. 75 (2016) 1978-1988.

[45] J.H. Lee, R. Durand, V. Gradinaru, F. Zhang, I. Goshen, D.S. Kim, L.E. Fenno, C. Ramakrishnan, K. Deisseroth, Global and local fMRI signals driven by neurons defined optogenetically by type and wiring, Nature 465 (2010) 788-792. 